\title{
Surface analysis by XRF and XPS for the Avogadro constant and the realization of the kilogram based on silicon spheres
}

Edyta Beyer, Michael Kolbe, Matthias Müller, Erik Darlatt

Physikalisch-Technische Bundesanstalt, Bundesallee 100, 38116 Braunschweig, Germany edyta.beyer@ptb.de, Fax: +49 53159269 1188, Tel: +49 5315921188

\section{Abstract}

The determination of the Avogadro constant for the redefinition of the SI unit kilogram as well as the eventual realization of the kilogram by monocrystalline enriched ${ }^{28} \mathrm{Si}$-spheres require a reliable surface analysis on silicon spheres. A novel instrumentation for the quantitative surface analysis of silicon spheres combines X-ray fluorescence spectroscopy and X-ray photoelectron spectroscopy techniques in order to enable the characterization and quantification of the oxide layer and unintentional contaminations. The analysis relies on the combination of the complementary X-ray methods. Measurements on ${ }^{28} \mathrm{Si}$-spheres were carried out. The surface was characterized regarding the elementary composition including the chemical binding states of the components and the mass of the surface layer

\section{Introduction}

Since the present definition of the SI unit kilogram is based on an artefact, which is the international prototype of the kilogram, there is an aim to redefine the unit kilogram in terms of fundamental constants. The future definition of the kilogram will contain the Planck constant $(h)$ whose numerical value has to be set by two independent experiments, and at least one with a relative measurement uncertainty of less than $2 \cdot 10^{-8}$. One way to define a value for the Planck constant is based on the Avogadro constant $\left(N_{A}\right)$. Therefore, an accurate measurement of $N_{A}$ also provides an accurate determination of the Planck constant [1].

For this reason the Avogadro constant is determined by "counting" the atoms in two isotopically enriched, monocrystalline ${ }^{28} \mathrm{Si}$-spheres with a mass of one kilogram. For the determination of $N_{A}$ the following equation is used:

$$
N_{A}=\frac{n \cdot M}{a^{3}} \cdot \frac{V}{m}
$$

With the molar mass $(M)$ and the lattice parameter $(a)$ of the silicon crystal, $\mathrm{n}=8$ being the number of atoms in a unit cell, the volume $(V)$ and the mass $(m)$ of the sphere [2].

Due to the always present surface layer on silicon, correction parameters have to be applied to the mass determination to achieve an accurate value for $N_{A}$. The surface layer is dominated by the silicon oxide layer but also consists of unintentional layers like carbonaceous contaminations and water (Figure 1). The mass of the surface layer has to be subtracted from the mass value of the sphere in order to obtain a small measurement uncertainty for the Avogadro constant. Thus it is necessary to measure the mass of the surface layer on silicon spheres.

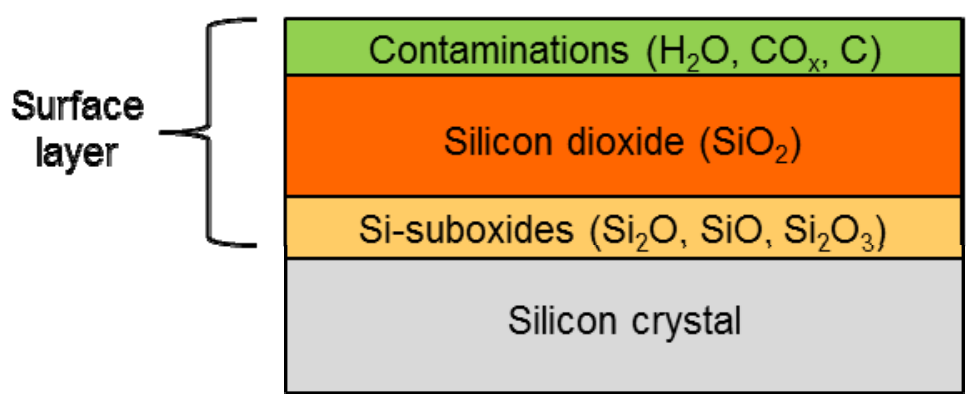

Figure 1 Model of the surface layer on silicon

In addition to the Avogadro constant the knowledge about the surface layer is also crucial for a future primary realization of the kilogram based on isotopically enriched, monocrystalline

${ }^{28} \mathrm{Si}$-spheres. The mass of the silicon core will then be determined by the number and mass 
of silicon atoms in the sphere whereto the mass of the surface layer has to be added to achieve the total mass value.

Previously the mass of the surface layer on silicon spheres has been determined with reference based X-ray fluorescence analysis using synchrotron radiation. The measurements were limited to only three areas on the sphere. Also the surface layer determination and the mass measurements were located in different cities, so it can be assumed that the surface layer, especially the carbonaceous contamination layer has been altered between the surface analysis and the mass measurements. Furthermore the assumption was made that all oxygen on the surface is bound in $\mathrm{SiO}_{2}$ and not in water or carbon layers [2].

To overcome the above mentioned barriers an instrumentation for combined X-ray analysis was set up to characterize and quantify the surface layer of silicon spheres with the opportunity to analyze every spot on the surface and link mass and surface measurements.

\section{Instrumentation}

The novel instrumentation provides combined measurements with $X$-ray fluorescence spectroscopy (XRF) and X-ray photoelectron spectroscopy (XPS) on spherical objects with diameters of about $93.7 \mathrm{~mm}$ (cf. figure 1). The analysis methods rely on the detection of fluorescence photons and photoelectrons respectively. Both are emitted by the sample surface as a consequence to excitation with X-rays. The excitation source is an Al Ka X-ray tube with a quartz crystal monochromator.

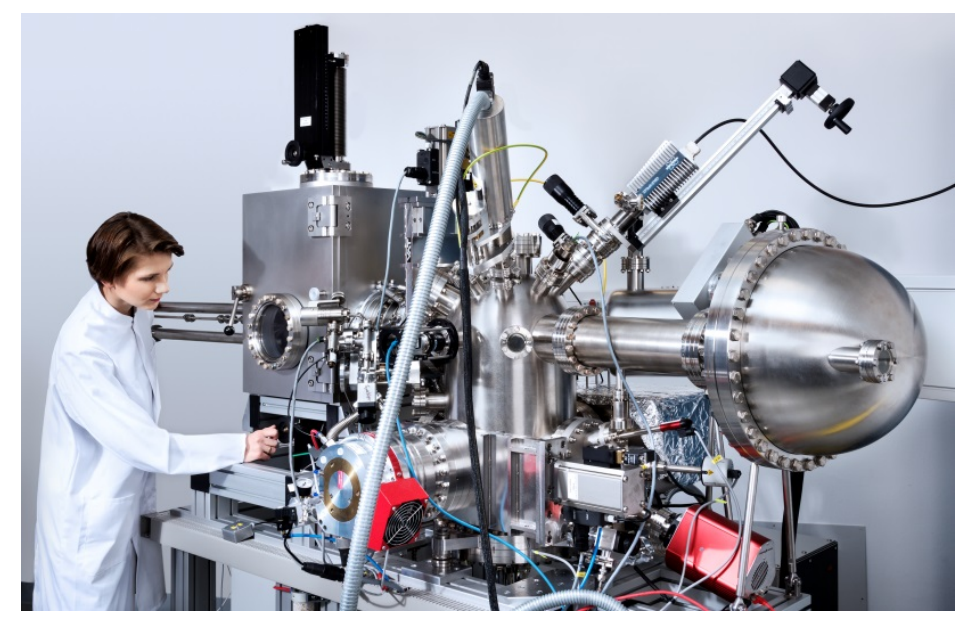

Figure 2 XRF/XPS-Instrumentation

To enable measurements on spherical samples, the ball manipulator inside of the measurement chamber is an essential part of the instrumentation. It allows measurements on every point on the sphere's surface and the change of the measurement geometry, especially the angle of incidence.

Beside spherical samples also flat samples can be measured in the instrumentation with the help of a mount for twelve samples.

Another uniqueness of the ultra-high vacuum instrumentation is its load lock that features a transport system allowing a sphere to move to a mass comparator under vacuum conditions. Thus surface analysis and mass determination can be carried out successively, with a lower risk of significant changes of the surface layer. The transfer is based on a portable vacuum container that is located in the load lock and can be attached to the load lock of a mass comparator as well.

\section{Surface analysis by combined XRF and XPS measurements}

The absolute quantification of the mass of the oxide layer is based on XRF measurements. The quantification approach relies on the correlation between the mass deposition of an element in an area to its fluorescence intensity [3]. A reference based quantification scheme is applied. Reference samples that were previously calibrated with X-ray reflectometry and reference free XRF with different thicknesses of silicon oxide layers are measured in the 
instrumentation with XRF. The ratio of the oxygen and the silicon signal from the calibration samples is used to derive a calibration curve to which the results of the sphere measurements are compared. By this way the mass deposition of oxygen on the surface of a sphere is obtained.

Figure 3 shows a fluorescence spectrum from a single point on the sphere and its fit of the model spectrum convoluted with detector response functions that were determined by a synchrotron radiation based calibration [4], fluorescence lines and as well different background contributions [5]. The count rates for the fluorescence lines of silicon and oxygen were determined from the deconvolution.

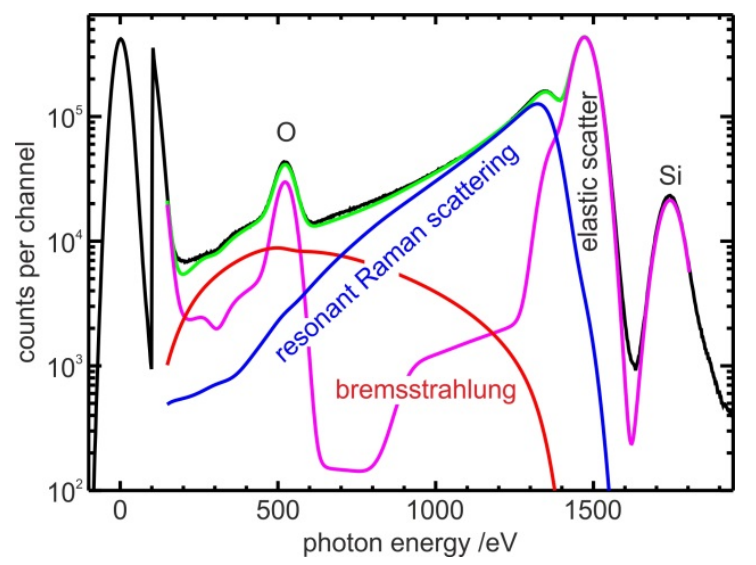

Figure $3 \mathrm{XRF}$-spectrum with Al Ka radiation from an X-ray tube

Due to the low resolution of the energydispersive fluorescence detector it is not possible to distinguish different contributions to a fluorescence line of an element. E.g. the oxygen peak is caused not only by oxygen bound in the silicon oxide layer but also from the oxygen in the contamination and water layer. A quantification of the oxide layer mass based only on XRF would lead to systematically higher mass values than the actual ones. This fact reasons the necessity of the XPS.

With the higher spectral resolution of the electron analyzer XPS allows the determination of the oxidation states of atoms and their assignment to different chemical species. Especially for the silicon atoms additional information about the silicon oxides on the sample surface is obtained. The photoelectron spectrum has a better resolution and smaller background contributions than the fluorescence spectrum, thus it allows an identification of elements in the sample. The survey spectra of the silicon sphere exhibit the elements silicon, carbon and oxygen, which are expected for a Si surface handled in ambient conditions. No further contamination was detected.

The detailed scan of the Si $2 p$ photoemission area exhibits the distribution of the silicon in the bulk, in the silicon dioxide layer, and in the various suboxides present at the interfaces (cf. figure 4).

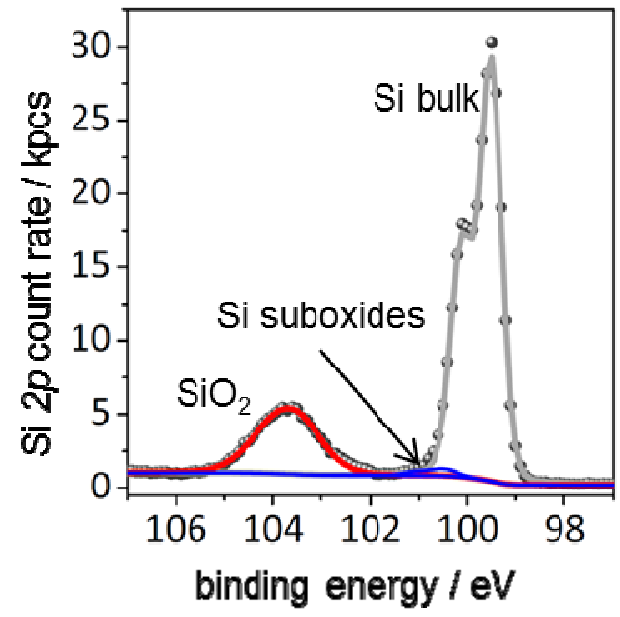

Figure 4 Spectrum of the Si2p photoemission 
With XPS the relative element concentration of each element in the surface can be determined. The relation of oxygen to the silicon oxide component of the Si $2 p$ peak shows, that there must be oxygen that is not bound in silicon oxide which agrees with the surface layer model mentioned above. The ratio of silicon oxide to oxygen is used as a correction factor for the mass deposition of oxygen gained through the XRF analysis. In this way the mass deposition of silicon oxide on a silicon sphere is determined by the combination of information from XRF and XPS.

\section{Results}

Exemplarily the results of the measurement of one ${ }^{28} \mathrm{Si}$-sphere are shown. The sphere was measured on 216 points on the surface with XRF and XPS. The measurements were carried out consecutively. All spectra were fitted.

With a Si/O-ratio of 0.43 the mass deposition for oxygen of $132.62 \mathrm{ng} / \mathrm{cm}^{2}$ and the surface area of the sphere the mass of the surface layer excluding the carbon is $64.1 \mu \mathrm{g}$ with a standard uncertainty of $6.7 \mu \mathrm{g}$. The mass of carbon has been derived by XPS only. Its value is $7.8 \mu \mathrm{g}$ (standard uncertainty $3.9 \mu \mathrm{g}$ ).

Without using the silicon to oxygen ratio obtained from the XPS the measurements and thus assuming that all oxygen is bound in the oxide layer would have led to a value for the surface layer of $68.6 \mu \mathrm{g}$ and a standard uncertainty of $6.7 \mu \mathrm{g}$.

\section{Conclusion}

The combined XRF/XPS-instrumentation allows the quantification of the surface layer on the whole sphere's surface avoiding the assumption that all oxygen is bound in the silicon oxide. The ratio of silicon in silicon dioxide to oxygen gives information about the distribution of oxygen between the oxide layer and the other unintentional layers like hydrocarbons and water. As shown in the results, the assumption, that all oxygen is bound in the silicon oxide layer would lead to a total mass of the surface layer that is about $4 \mu \mathrm{g}$ bigger than the real value.

Owing to the vacuum transfer system the analysis can be linked to mass measurements without altering the surface layer. Several measurement cycles of mass and surface determination under vacuum conditions can be carried out.

Surface analysis on silicon spheres decreases the measurement uncertainty of the Avogadro and Planck constants and, corresponding to this, a realization of the kilogram via silicon spheres can be improved. In further investigations also the long term stability of different silicon spheres will be analyzed to gain knowledge about the long term behavior of spheres as mass standards, as changes of the spheres are expected predominantly in the surface layer.

\section{References}

[1] Andreas B, Azuma Y, Bartl G, Becker P, Bettin H, Borys M, Busch I, Fuchs P, Fujii K, Fujimoto $\mathrm{H}$, Kessler E, Krumrey M, Kuetgens U, Kuramoto N, Mana G, Massa E, Mizushima S, Nicolaus A, Picard A, Pramann A, Rienitz O, Schiel D, Valkiers S, Waseda A, Zakel S 2011 Counting the atoms in a ${ }^{28} \mathrm{Si}$ crystal for a new kilogram definition Metrologia 48 1-13

[2] Busch I, Azuma Y, Bettin H, Cibik L, Fuchs P, Fujii K, Krumrey M, Kuetgens U, Kuramoto N and Mizushima S 2011 Surface layer determination for the Si spheres of the Avogadro project Metrologia 48(2), 62-82

[3] Kolbe M, Beckhoff B, Krumrey M, Ulm G 2005 Thickness determination for $\mathrm{Cu}$ and $\mathrm{Ni}$ nanolayers: Comparison of reference-free fundamental-parameter based X-ray fluorescence analysis and X-ray reflectometry Spectrochimica Acta B 60 505-510

[4]Beckhoff B 2008 Reference-free X-ray spectrometry based on metrology using synchrotron radiation J. Anal. At. Spectrom. $23845-853$

[5] Müller M, Beckhoff B, Ulm G, Kanngießer B 2006 Absolute determination of cross sections for resonant Raman scattering on silicon Phys. Rev. A 74, 012702 